\title{
Re-Examining the "Out of Africa" Theory and the Origin of Europeoids (Caucasoids) in Light of DNA Genealogy
}

\author{
Anatole A. Klyosov ${ }^{*}$, Igor L. Rozhanskii \\ The Academy of DNA Genealogy, Newton, USA \\ Email: "aklyosov@comcast.net
}

Received January $7^{\text {th }}, 2012$; revised February $4^{\text {th }}, 2012$; accepted March $10^{\text {th }}, 2012$

\begin{abstract}
Seven thousand five hundred fifty-six (7556) haplotypes of 46 subclades in 17 major haplogroups were considered in terms of their base (ancestral) haplotypes and timespans to their common ancestors, for the purposes of designing of time-balanced haplogroup tree. It was found that African haplogroup A (originated $132,000 \pm 12,000$ years before present) is very remote time-wise from all other haplogroups, which have a separate common ancestor, named $\beta$-haplogroup, and originated $64,000 \pm 6000 \mathrm{ybp}$. It includes a family of Europeoid (Caucasoid) haplogroups from $F$ through $T$ that originated 58,000 $\pm 5000 \mathrm{ybp}$. A downstream common ancestor for haplogroup $\mathrm{A}$ and $\beta$-haplogroup, coined the $\alpha$-haplogroup emerged $160,000 \pm 12,000 \mathrm{ybp}$. A territorial origin of haplogroups $\alpha$ - and $\beta$-remains unknown; however, the most likely origin for each of them is a vast triangle stretched from Central Europe in the west through the Russian Plain to the east and to Levant to the south. Haplogroup B is descended from $\beta$-haplogroup (and not from haplogroup A, from which it is very distant, and separated by as much as 123,000 years of "lateral" mutational evolution) likely migrated to Africa after 46,000 ybp. The finding that the Europeoid haplogroups did not descend from "African" haplogroups A or B is supported by the fact that bearers of the Europeoid haplogroups, as well as all non-African haplogroups do not carry either SNPs M91, P97, M31, P82, M23, M114, P262, M32, M59, P289, P291, P102, M13, M171, M118 (haplogroup A and its subclades SNPs) or M60, M181, P90 (haplogroup B), as it was shown recently in "Walk through Y" FTDNA Project (the reference is incorporated therein) on several hundred people from various haplogroups.
\end{abstract}

Keywords: Y Chromosome; Mutations; Haplotypes; Haplogroups; TMRCA; STR; SNP; "Out of Africa"

\section{Introduction}

This study concerns the origin of anatomically modern humans, which presumably belong to Y chromosomal haplogroups A through $\mathrm{T}$ according to the classification developed in human genetics and DNA phylogeny of man. This paper 1) sets forth a timeframe for the origin of Europeoids (Caucasoids); 2) identifies their position among all haplogroups (tribes) known today on the haplogroup tree; and 3) offers evidence to re-examine the validity of the "Out of Africa" concept.

The principal difference of our approach from those known in human genetics is that our methodology is based on the identification of branches of haplotypes in each haplogroup and its subclade (each branch is descended from its only common ancestor), and, in each case, is calculated a timespan from a common ancestor of the branch by verifying that the branch is indeed derived from one common ancestor and by using the criteria described in (Klyosov, 2009a; Rozhanskii \& Klyosov, 2011; Rozhanskii, 2011). As a result, we obtained a chronology of all available branches in each haplogroup and in their total entirety-from $A$ to $T$ (in the current classification). In other words, for each haplotype we successfully identified its place in the whole multi-haplogroup system of mankind. It is reasonable to assume that haplotypes of the whole of mankind form a continuous system, albeit locally interrupted by "population bottlenecks" which essentially disrupt the initially continuous fab-

${ }^{*}$ Corresponding author. ric of haplotypes. This fabric can be reconstructed based on its fragments and in the same manner as the kinetics of chemical reactions can be reconstructed based on relatively few experimental points. This analogy is rather close since mutations in haplotypes obey the same laws of chemical kinetics, this was discussed in the first paper of this series (Rozhanskii \& Klyosov, 2011).

Thanks largely in part to geneticists, the "Out of Africa" concept was popularized during the last two decades, yet it was never directly proven; however, for many specialists its appeal was undeniably convincing. The concept was based primarily on the premise that Africa possesses the highest variability, or variance, of the human DNA and its segments. Set apart, it is not a strong argument because a mix of different DNA lineages also results in a high variability and, as we show below, it is largely what occurs in Africa. Moreover, a genomic gap exists between some Africans and non-Africans, which has also been interpreted as an argument that the latter descended from Africans. A more plausible interpretation might have been that both current Africans and non-Africans descended separately from a more ancient common ancestor, thus forming a proverbial fork. A region where this downstream common ancestor arose would not necessarily be in Africa. In fact, it was never proven that he lived in Africa.

Research into this question has served as the basis for and the subject of our work. We have found that a great diversity of $Y$ chromosomal haplotypes in Africa is a result of the mixing of 
several very distant lineages, some of them not necessarily African, and that Europeiods (at least) do not contain "African" SNPs (those of haplogroups A or B). These important findings put a proverbial dent in the "Out of Africa" theory.

\section{Results and Discussion}

The 22 marker haplotypes, which are the "slowest" in terms of their mutation rate constant, described in (Klyosov, 2011a, 2011b; Rozhanskii \& Klyosov, 2011) were mainly used in this study. They are best suited for chronological calculations down to 100,000 years and deeper in time. This is because one mutation in these haplotypes occurs on average once in 4250 years, while in 67 marker haplotypes, for example, one mutation occurs - on average - once in 208 years (ibid). However, the 22 marker haplotypes include a part of the last panel of the 67 marker haplotypes and hence, 67 marker haplotypes were needed for the study.

\section{Haplogroup A}

Extended haplotypes of haplogroup A collected in various databases (YSearch, FTDNA Projects), split into at least four different and distinctive DNA lineages, each with its base haplotype. This is essentially represented with a series of 32 marker haplotypes (Figure 1) and 37 marker haplotypes (Figure 2).

In a simple, uncomplicated case, the base haplotype is equivalent to the ancestral haplotype in the lineage. This is obvious in recent lineages, in which most haplotypes still represent the non-mutated ancestral haplotype. For more ancient lineages the

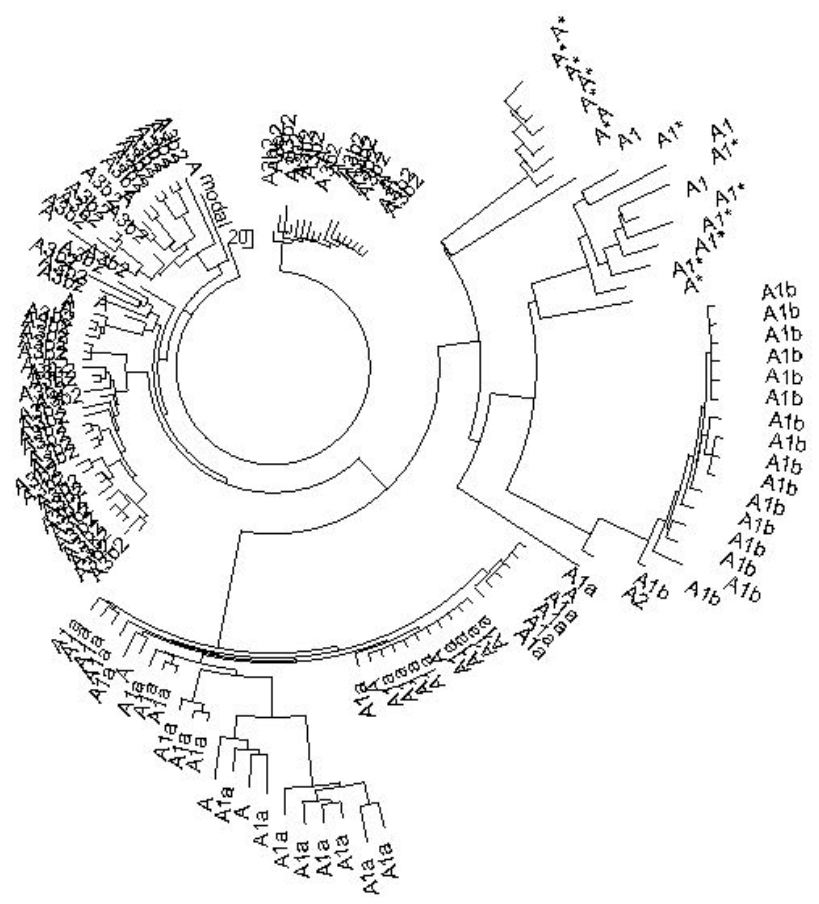

Figure 1.

A tree of 141 of 32 marker haplotypes of haplogroup A. Haplotypes were taken from SMGF and FTDNA's "Y-Haplogroup A Project" (http://www.familytreedna.com/public/Haplogroup_A/default.aspx?sect ion=yresults). A series of previously unreported haplotypes from Cameroon was assigned to Alb subclade according to STR values, being nearly identical to those found in Bahamas (Simms et al., 2011).

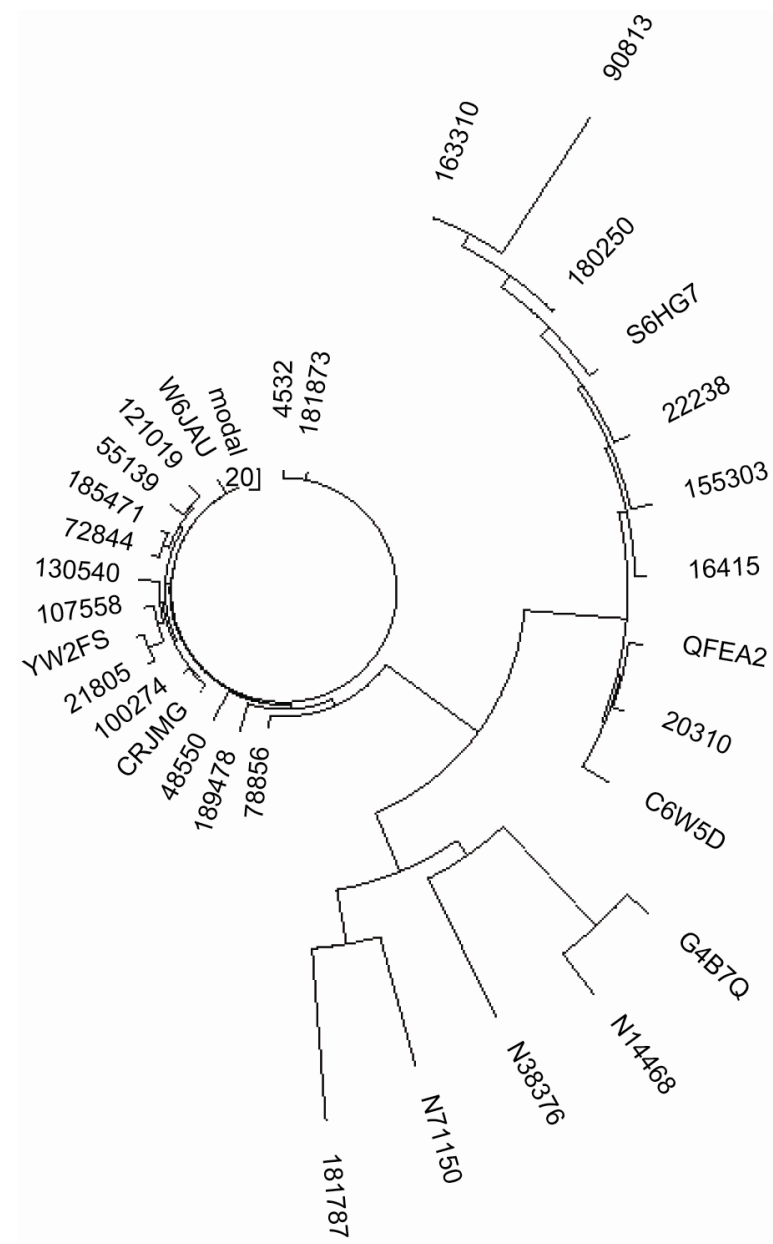

Figure 2.

A tree of 31 of 37 marker haplotypes of haplogroup A with some subclades. Haplotypes were taken from YSearch и FTDNA's "African Project"

(http://www.familytreedna.com/publicwebsite.aspx?vgroup=Africa n.DNAProject\&section=yresults).

base haplotype is obtained by the minimization of mutations in the haplotype dataset; therefore, the base haplotype represents the deduced ancestral haplotype. The four base haplotypes of haplogroup $\mathrm{A}$ in the 22 marker format are as follows:

$$
\begin{aligned}
& 121111-911-10-10912127108 \text { null } 13111610 \\
& 1491111 \\
& 121011-713-8-1081517610912131116813 \\
& 111112 \\
& 131112-1011-16-10914148889121112812 \\
& 121111 \\
& 121310-1011-10-1181515898 \text { null } 10914812 \\
& 81112
\end{aligned}
$$

These base haplotypes have been assigned to subclades A3b2, A1a, A* (P97+, SRY10831.1-), and A* (M23-, M32-, P108-, SRY10831.1-), respectively. It was calculated that the common ancestors of the branches lived 5500, 5000, 600 years before present (ybp), and the last one is an individual haplotype. It is clear that these four haplotypes are tremendously distant from each other, and this is taking into account the extremely low mutation rates of the markers. The deduced base haplotype for 
haplogroup A from the available data is as follows:

$121111-911-10-1081415710812131116813$ 91112

Since alleles in the four haplotypes above vary significantly, the permutation method was applied (Klyosov, 2009a) for calculation of a timespan to their common ancestor. The average squared sum of all mutations in the four haplotypes above equals to 984 , and the number of conditional generations to a common ancestor is $984 / 22 / 2 / 16 / .00027=5177$. That is, 132,000 years to a common ancestor of all the available haplotypes of haplogroup A. In the formula above, 22 is the number of markers in each haplotype; sixteen (16) is the square of the number of haplotypes in the dataset, 2 is introduced because the number of mutations was counted twice (all permutations), and .00027 is the mutation rate constant per marker in the 22 marker haplotypes (Klyosov, 2011a, 2011b). A correction for back mutations is not required in the permutation method (Klyosov, 2009a).

Pairwise calculations of the dataset above give, as it should be, slightly lower values of timespans to a common ancestor. For example, base haplotypes of the A1a and A3b2 subclades (see Figure 2) differ by 25 mutations in all 22 markers, which places their common ancestor at $4167 \rightarrow 8576$ conditional generations, that is $112,000 \mathrm{ybp}$. Two haplotypes with nullmutation differ by 27 mutations, which places their common ancestor at $4500 \rightarrow 9922$ conditional generations, that is 127,000 years to a common ancestor (see Materials and Methods for the principles of calculations). This gives an additional support of the obtained "age" of haplogroup A as 132,000 \pm 20,000 years.

\section{Haplogroup B}

A similar approach was applied to haplogroup B, and the following 22 marker base haplotype was obtained for a common ancestor who lived 46,000 ybp (Klyosov, 2011b):

$111211-1111-10-1181616810812101115812$ 111211

It differs from the base 22 marker haplotype of haplogroup A by 18 mutations, which gives $18 / .006=3000$ generations. With a correction factor (for back mutations) of 1.633, the result constitutes 123,000 years between common ancestors of haplogroup A and B. Because they lived 132 and 46 thousand years before present, respectively, their common ancestor lived approximately 150,000 years before present (see Materials and Methods).

The ISOGG (International Society of Genetic Genealogy) annual review in 2010 and earlier stated "The BR haplogroup split off from haplogroup A 55,000 years before present (bp). It probably appeared in North East Africa". Since the ISOGG2012 stated, "The A haplogroup is thought to have been defined about 60,000 years bp"

http://www.isogg.org/tree/ISOGG_YDNATreeTrunk.html then haplogroups A and B should be separated by only several thousand years, or by $2-3$ mutations in their slow 22 marker base haplotypes. It is not so, and between their base haplotypes there are as many as 18 mutations in the 22 markers, which translates to 123 thousand years (see above).

This finding indicates that haplogroup B did not descend from haplogroup A. Rather, they both descended from a com- mon ancestor who lived $\sim 150,000 \mathrm{ybp}$, and he was not necessarily living in Africa. Since he belonged to a haplogroup upstream from haplogroups $\mathrm{A}$ and $\mathrm{B}$, his haplogroup can be named "alpha-haplogroup". It is a matter of taste and belief to call it "Adam" or not.

\section{Haplogroups $\mathrm{C}$ through $\mathrm{T}$}

The same methodology was applied to 7415 of 67 marker haplotypes of all known haplogroups and their subclades, reduced to the slow 22 marker haplotypes, taken from databases YSearch and SMGF and a multitude of FTDNA Projects (see Appendix). The base haplotypes of principal haplogroups and some of their subclades, including those of haplogroups A and $\mathrm{B}$, are listed below, and chronology of their appearance is shown in Figure 3.

$121111-911-10-1081415710812131116813$

91112

$111211-1111-10-1181616810812101115812$

111211

$111011-1111-10-1181616810812101115812$

$11 \quad 1211$

(B2a1)

$111311-1111-11-1081616810812121113812$

$\begin{array}{lllll}10 & 11 & 11 & \left(\mathrm{C}^{*}\right)\end{array}$

$111311-1111-10-1081616810812121213812$

$11 \quad 1111$

$111311-1112$ - 10 - 1091616810812111213812

$1211 \quad 10$

$111211-1111-9-1081516810812111212712$

121111

$111211-1011-10-1081618810812111215712$

101111

$11127-1111-10-1081616810812121212713$

121111

(D3a)

$111211-1111-10-1081515810812121212812$

$11 \quad 1211$

$111211-1111-9-1081515810812111212812$

121211

$111211-1111-10-1081516810812111212713$

$12 \quad 11 \quad 11$

(E1b1)

$111211-1011-10-111015168108121112128$

$1411 \quad 1112$

111211 - 1111 - 10 - 1191516810812111212813

$11 \quad 11 \quad 12$

$111211-1111-10-1181516810812101212813$

$11 \quad 1111$

$111211-1111-10-1181516810814101212813$

111111

$111211-1111-10-1181616810811101112813$

$11 \quad 1111$

$111211-912-10-1181616810813101212812$

111214

$111211-1012-9-1181616810813101212812$

111214

$111411-1111-10-1181516810812101212813$

$1211 \quad 12$

$111411-811-10-1181515810812101212813$

111112

$111311-1111-10-1181516810812111212813$

$\begin{array}{lll}10 & 12 & 12\end{array}$

$111311-1111-10-1281616810812111213813$ 
$\begin{array}{lll}12 & 12 & 12\end{array}$

$\left(\mathrm{I} 2^{*}\right)$

$111311-1012$ - $10-1181516810812111212813$

$12 \quad 12 \quad 12$

(I2b2)

$111611-1111-10-1181516810812101212812$

$11 \quad 12 \quad 12$

111511 - 1111 - 9 - 1171515810812101212812

$\begin{array}{lll}11 & 12 & 12\end{array}$

$111214-1112-10-1181517710813111212812$

$11 \quad 12 \quad 12$

$111213-1111-10-118151787812111212712$

111112

(NO)

$111212-1112-10-1181516810812101212712$

$11 \quad 11 \quad 12$

(N1b)

$111214-1112-10-1181517810812101212712$

$11 \quad 11 \quad 12$

(N1c1)

111213 - 1111 - 10 - 119151787812111212712

111112

121212 - $1111-11-1181517810812101212812$

$\begin{array}{lll}11 & 11 & 12\end{array}$

$121214-1111-11-1181517810812111212812$

111112

(Q1)

121212 - $1111-11-1181517810812101212812$

$\begin{array}{lll}11 & 11 & 12\end{array}$

$121213-1111-11-1181616810812101212812$

$\begin{array}{lll}11 & 11 & 12\end{array}$

(R1a)

$121211-1111-11-1181717810812101212812$

$11 \quad 1112$

(R1a1a1)

$121313-1111-11-1181516810812101212811$

$\begin{array}{lll}11 & 11 & 12\end{array}$

(R1b1)

$121210-1111-11-1181517810812101212812$

$\begin{array}{lll}11 & 12 & 13\end{array}$

$111213-1113-9$ - 1181717810812101212811

121112

(T1a)

$111213-1112-9-1181717810812101212811$

121112

(T1b)

$111211-1111-10-1181516810812101212812$

$11 \quad 11 \quad 12$

( $\beta$-haplogroup)

Pairwise calculations of mutation distances between the base haplotype of haplogroup A and each of the base haplotypes of other haplogroups place a common ancestor of the $\alpha$-haplogroup at $160,000 \pm 12,000$ years before present. For example, $18 \mathrm{mu}-$ tations between A and B base haplotypes, as it was described above, result in 150,000 ybp for their common ancestor. Twentyone (21) mutations with haplogroup DE base haplotype give $167,000 \mathrm{ybp}$ for their common ancestor. Twenty-three (23) mutations with haplogroup $\mathrm{H}$ base haplotype result in 171,000 ybp for their common ancestor with haplogroup A. For haplogroup I (21 mutations) it is 161,000 ybp. For haplogroup Q (22 mutations) it is $166,000 \mathrm{ybp}$. For haplogroup $\mathrm{R}$ (21 mutations) it is $160,000 \mathrm{ybp}$. The distance in 19 mutations between the base haplotypes of haplogroup A and $\beta$-haplogroup places $\alpha$-haplogroup at $165,000 \mathrm{ybp}$. Clearly, the base haplotype of haplogroup A and its subclades is very remote from all other haplogroups.

Similar pairwise calculations with the base haplotype of haplogroup B as well with all other haplogroups (besides A) place a common ancestor of beta-haplogroup to $64,000 \pm 6000$ years before present (see Figure 3). This haplogroup is close to or identical with the BT haplogroup according to the current classification.

Figure 3 shows a topology of the current haplogroup tree.
The $\alpha$-haplogroup which is the ancestral one with respect to both African (left branch) and non-African haplogroups (right branch) arose around 160,000 ybp, and 132,000 ybp gave rise to haplogroup A. Another, quite different branch, had formed a fork, then apparently went through a population bottleneck around 70 - 60 thousand ybp (perhaps the Toba event), and gave rise to $\beta$-haplogroup, ancestral to non-African haplogroups, $64,000 \pm 6000 \mathrm{ybp}$.

Apparently, haplogroup B was initially not of an African origin. It could have migrated to Africa and mixed there with a local Negroid population. A common ancestor of the presentday bearers of haplogroup B lived 46,000 ybp. A similar story had occurred with a group of bearers of haplogroup R1b1 around $4000 \mathrm{ybp}$, who ventured to the center of African continent during their westward migration along the African Mediterranean shore, an became a Negroid population having an unusual (for Africa) haplogroup (Cruciani et al., 2010; Klyosov, 2012).

The Mongoloid and Austronesian haplogroup C split $~ 36,000$ ybp and gradually populated regions of Central Asia, Australia and Oceania. Haplogroup DE split to D and E around 42,000 $\mathrm{ybp}$, and currently populates vast territory from North Africa to the west to Korea and Japan to the east.

The family of haplogroup from $\mathrm{F}$ through $\mathrm{T}$ is largely the Europeoid (Caucasoid) family. Most of bearers of these haplogroups remained Europeoids; however, some populations have acquired racial features of the prevailing races in a given region, recently or in the long past.

Based on the calculations given in this study, we know that the far most bearers of haplogroup A live in Africa, and they lived there probably all or most of those 132,000 years since haplogroup A arose. It cannot be excluded, of course, that haplogroup A might have been appeared elsewhere and then migrated to Africa. However, there is no reason to believe (and fewer reasons to insist) that the Europeoid family originated in Africa.

\section{Lack of the African SNP (Haplogroup A) in Non-Africans}

A critical datapoint has emerged that disproves the "Out of Africa" concept; specifically, recent data shows that non-African people have neither M91, P97, M31, P82, M23, M114, P262, M32, M59, P289, P291, P102, M13, M171, M118 (haplogroup A and its subclades SNPs), nor M60, M181, P90 (haplogroup B SNPs) in their Y-chromosomes.

In fact, according to the data obtained from the "Walk Through the Y" (chromosome) international project conducted by Family Tree DNA (Texas and Arizona) [see Appendix] not one non-African participant out of more than 400 individuals in the Project tested positive to any of thirteen "African" subclades of haplogroup A, SNPs for which indicated above. If to take, for example, bearers of R1a haplogroup, they each have the ladder of SNPs from M42 and M139 (haplogroup BT, but not haplogroup B, which, as it was described above, split and migrated to Africa around 46,000 ybp or earlier), through M168 and M294 (haplogroup CT), P143 (haplogroup CF), M89 and P158 (haplogroup F), L15 and L16 (haplogroup IJK), M9 (haplogroup K), M74, L138, P69, P230, P243, P244, P280, P284, P286 (haplogroup P), M207, P224, P227, P229, P232, P280, P285 (haplogroup R), P231, P241, P242, P245, P294 (haplogroup R1), L145 and L146 (haplogroup R1), L120 and 


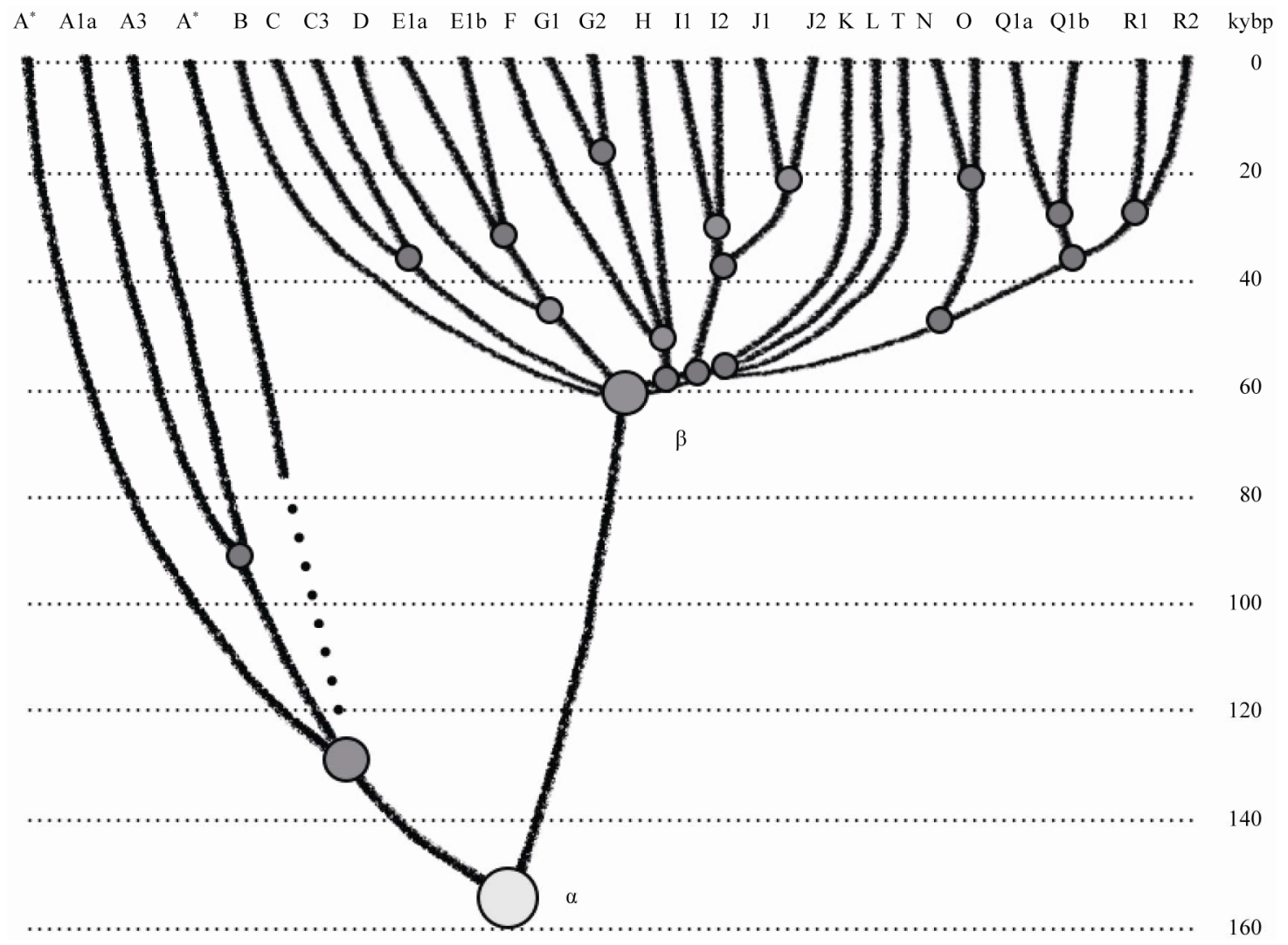

Figure 3.

A haplogroup tree of Y chromosome derived from base haplotypes of haplogroups and subclades and their TMRCAs, systematically calculated as described in this study. 7415 haplotypes from 46 subclades of 17 major haplogroups have been considered for the tree design. Timescale on the vertical axis shows thousands of years from the common ancestors of the haplogroups and subclades. The tree shows the alpha-haplogroup, which is the ancestral haplogroup of the African and non-African haplogroups, and the beta-haplogroup, which is the ancestral haplogroup, close or identical with BT haplogroup in the current classification. The left branch represents haplogroup A (arose $\sim 132,000 \mathrm{ybp}$ ) and its subclades. The right branch of haplogroups $\mathrm{F}$ through $\mathrm{R}$ including T) represent Europeoids (Caucasoids) arose $\sim 58,000$ years before present. Haplogroup B (arose $\sim 46,000$ ybp) migrated to Africa, the Mongoloid and Austronesian haplogroup C split 36,000 ybp, apparently Middle Eastern haplogroups DE split $\sim 42,000$ ybp. A region of the origin of the alpha-haplogroup $\sim 160,000$ ybp remains unknown. The Europeoid family of haplogroups arose apparently in the triangle between Central Europe on the west, the Russian Plain (Eastern European Plain) on the east and Levant on the south.

L122 (haplogroup R1a1), L168 (haplogroup R1a1a). In other words, all of the SNP have been identified, which should be found according to the phylogeny of R1a, but SNPs of the all examined subclades of haplogroup A were completely absent. The same pattern was observed with all other bearers of nonAfrican haplogroups. The bearers of haplogroup A were exclusively positive to M91 SNP, characteristic of that haplogroup.

There are, however, four distinct SNPs which present in both Africans and Europeans of haplogroup R1a1, taken the latter as an example. They seem to be the most ancient SNPs, which are defined the alpha-haplogroup (see Figure 3). Tables 1 and 2 illustrate this statement.

The ancestral alleles of the above four SNPs should correspond to the alpha haplogroup. All four are mutated in haplogroup R1a1, and the WTY data show. All four are still ancestral in the A1 subclade. All other subclades of haplogroup A show various combinations of the SNPs which do not match those in haplogroup R1a1 (see also Table 2).

Table 2 shows SNPs of five subclades of "African" haplogroup A. None of those SNPs have been observed in hap-
Table 1.

List of SNPs identified in haplogroup R1a1 and subclades of haplogroup A. Ancestral and derived alleles are shown. For blank spaces data are not available. Cont. in Table 2.

\begin{tabular}{|c|c|c|c|c|c|c|}
\hline SNP & R1a1 & $\mathrm{A} 1$ & A1a & $\mathrm{A} 1 \mathrm{~b}$ & A2 & $\mathrm{A} 3 \mathrm{~b} 2$ \\
\hline \multicolumn{7}{|c|}{ V168 (alpha) } \\
\hline $\mathrm{G} \rightarrow \mathrm{A}$ & A & G & A & & & \\
\hline \multicolumn{7}{|c|}{ V171 (alpha) } \\
\hline $\mathrm{C} \rightarrow \mathrm{G}$ & G & $\mathrm{C}$ & G & & G & \\
\hline \multicolumn{7}{|c|}{ V221 (alpha) } \\
\hline $\mathrm{G} \rightarrow \mathrm{T}$ & $\mathrm{T}$ & $\mathrm{G}$ & G & & $\mathrm{T}$ & \\
\hline \multicolumn{7}{|c|}{ P108 (alpha) } \\
\hline $\mathrm{C} \rightarrow \mathrm{T}$ & $\mathrm{T}$ & $\mathrm{C}$ & $\mathrm{C}$ & $\mathrm{C}$ & $\mathrm{T}$ & $\mathrm{T}$ \\
\hline
\end{tabular}

logroup R1a1, it maintains their ancestral state.

These data, based on the SNPs ( Single Nucleotide Polymor-

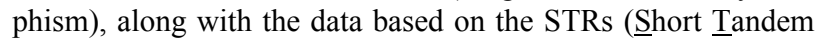


Table 2.

List of alleles of "African" SNPs identified in haplogroup R1a1 and subclades of haplogroup A. Cont. from Table 1.

\begin{tabular}{|c|c|c|c|c|c|c|}
\hline SNP & R1a1 & $\mathrm{A} 1$ & Ala & $\mathrm{A} 1 \mathrm{~b}$ & A2 & $\mathrm{A} 3 \mathrm{~b} 2$ \\
\hline \multicolumn{7}{|c|}{ M31 (A1a) } \\
\hline $\mathrm{G} \rightarrow \mathrm{C}$ & G & & $\mathrm{C}$ & & & G \\
\hline \multicolumn{7}{|c|}{ V50 (A2) } \\
\hline $\mathrm{T} \rightarrow \mathrm{C}$ & $\mathrm{T}$ & $\mathrm{T}$ & & & $\mathrm{C}$ & \\
\hline \multicolumn{7}{|c|}{ M32 (A3) } \\
\hline $\mathrm{T} \rightarrow \mathrm{C}$ & $\mathrm{T}$ & $\mathrm{T}$ & G & & $\mathrm{T}$ & $\mathrm{C}$ \\
\hline \multicolumn{7}{|c|}{ P289 (A3b) } \\
\hline $\mathrm{C} \rightarrow \mathrm{G}$ & $\mathrm{C}$ & & & & & \\
\hline \multicolumn{7}{|c|}{ M13 (A3b2) } \\
\hline $\mathrm{G} \rightarrow \mathrm{C}$ & G & & & & & $\mathrm{C}$ \\
\hline
\end{tabular}

Repeats), described in this study, are compatible with each other and undeniably indicate that non-African people, bearers of haplogroups from C to T, did not descend from the "African" haplogroups A or B. Their origin is likely not in Africa. A higher variance of the DNA in Africa, which was a cornerstone of the "Out of Africa" theory, is explained by Figure 3, in which haplogroup A has been evolving (mutation-wise) for 132,000 years, while the non-European haplogroups are much younger. Hence, there is a lower variability in the latter. The same is related to language variability, which has also been used as an argument of the African origin of non-Africans. We believe that those arguments upon which the "Out of Africa" theory was based were, in fact, conjectural, incomplete and not actually data-driven. Therefore, we are left holding the question of the origin of Homo sapiens.

Based on palaeoarchaeological evidence, the region, where anatomically modern humans have likely originated, is comprised of a vast territory from Central Europe in the west to the Russian Plain in the east to Levant in the south. Each of these regions is renowned for discoveries of the oldest skeletal remains of modern humans dating back to 42,000 - 44,000 ybp. To date, none of these sub-regions has clear and unequivocal advances in this regard.

\section{Materials and Methods}

7556 haplotypes, predominantly 67 marker ones, have been collected in databases FTNDA, YSearch and SMGF (Sorenson Database), and reduced to the slow 22 marker haplotype panels:

DYS426, DYS388, DYS392, DYS455, DYS454, DYS438, DYS531, DYS578, DYS395S1a, DYS395S1b, DYS590, DYS641, DYS472, DYS425, DYS594, DYS436, DYS490, DYS450, DYS617, DYS568, DYS640, DYS492.

The methodology of haplotype datasets analysis was described in (Klyosov, 2009a, 2009b; Rozhanskii \& Klyosov, 2011). The most important research component involved dissecting the dataset to branches of haplotypes, each branch descended from one common ancestor. This was examined and verified by the logarithmic method (no mutation counting) coupled with the linear method (based on mutation counting), as described in (Klyosov, 2009a; Rozhanskii, 2011). The mutation rate constant for the 22 marker haplotypes equals to $.0060 \mathrm{mu}-$ tation/haplotype/conditional generation of 25 years, or .00027 mutation/marker/generation (Klyosov, 2011a; Rozhanskii \& Klyosov, 2011). Haplotype trees were composed using software PHYLIP, Phylogeny Inference Package program (see Klyosov, 2009a, 2009b and references therein). Corrections for back mutations were introduced as described in (Klyosov, 2009a; Rozhanskii \& Klyosov, 2011). Margins of error were calculated as described in (Klyosov, 2009a). Permutation method of TMRCA (time to the most recent common ancestor) calculation was described in (Klyosov, 2009a).

Base haplotypes in the dataset were determined by minimization of mutations; by definition, the base haplotype is one which has the minimum collective number of mutations in the dataset. The base haplotype is the ancestral haplotype or the closest approximation to the latter.

Example: Calculation of a timespan to a common ancestor for the base haplotype for haplogroup A $(132,000 \mathrm{ybp})$

$$
\begin{aligned}
& 121111-911-10-1081415710812131116813 \\
& 91112 \\
& \text { (A) }
\end{aligned}
$$

and that for the $\beta$-haplogroup $(64,000 \mathrm{ybp})$

$$
111211-1111-10-1181516810812101212812
$$$$
11 \quad 11 \quad 12
$$

( $\beta$-haplogroup)

19 mutations between the two base haplotypes results in $19 / .006=3167$ conditional generations $(25$ years each $)$ without a correction for back mutations. The number of mutations per marker is $19 / 22=.8636$. By employing formula for back mutations (Klyosov, 2009a), we find that a correction for back mutation is

$$
\frac{1}{2}(1+\exp (.8636))=1.686
$$

Therefore, the "lateral" time difference between two base haplotypes is $3167 \times 1.686=5340$ conditional generations of 25 year, that is 133,500 years. A common ancestor of the both base haplotypes, $A$ and $\beta$-haplotype, lived $(133,500+64,000+$ $132,000) / 2=164,750$ ybp.

Assignments of haplotypes to haplogroups and subclades were based on their SNP classification, as provided in the databases. In some instances it was additionally supported by calculating their position of the phylogenic trees from their respective STR data.

\section{Acknowledgements}

The authors are indebted to Dr. Alexander Zolotarev, a participant of the WTY project, for providing data of the Project, and to Ms. Laurie Sutherland for valuable help with the preparation of the manuscript.

\section{REFERENCES}

Cruciani, F., Trombetta, B., Sellitto, D., Massaia, A., Destro-Bisol, G., Watson, E., et al. (2010). Human Y chromosome haplogroup R-V88: A paternal genetic record of early mid Holocene trans-Saharan connections and the spread of Chadic languages. European Journal of Human Genetics, 18, 800-807.

Cruciani, F., Trombetta, B., Massaia, A., Destro-Bisol, G., Sellitto, D., \& Scozzari, R. (2011). A revised root for the human Y chromosomal phylogenetic tree: The origin of patrilineal diversity in Africa. The American Journal of Human Genetics, 88, 1-5. doi:10.1016/j.ajhg.2011.05.002

Klyosov, A. A. (2009a). DNA Genealogy, mutation rates, and some 


\section{A. A. KLYOSOV, I. L. ROZHANSKII}

historical evidences written in Y-chromosome. I. Basic principles and the method. Journal of Genetic Genealogy, 5, 186-216.

Klyosov, A. A. (2009b). DNA Genealogy, mutation rates, and some historical evidences written in Y-chromosome. II. Walking the map. Journal of Genetic Genealogy, 5, 217-256.

Klyosov, A. A. (2011a). The slowest 22 marker haplotype panel (out of the 67 marker panel) and their mutation rate constants employed for calculations timespans to the most ancient common ancestors. Proceedings of the Russian Academy of DNA Genealogy, 4, 1240-1257.

Klyosov, A. A. (2011b). DNA genealogy of major haplogroups of Y chromosome (Part 1). Proceedings of the Russian Academy of DNA Genealogy, 4, 1258-1283.

Klyosov, A. A. (2012). Ancient history of the Arbins, bearers of hap- logroup R1b, from Central Asia to Europe, 16,000 to 1,500 years before present. Advances in Anthropology, in press.

Rozhanskii, I. (2010). Evaluation of the convergence of sets in STR phylogeny and analysis of the haplogroup R1a1 tree. Proceedings of the Russian Academy of DNA Genealogy, 3, 1316-1324.

Rozhanskii, I. L., \& Klyosov, A. A. (2011). Mutation rate constants in DNA genealogy (Y chromosome). Advances in Anthropology, 1, 2634. doi:10.4236/aa.2011.12005

Simms, T. M., Martinez, E., Herrera, K. J., Wright, M. R., Perez, O. A., Hernandez, M. et al. (2011). Paternal lineages signal distinct genetic contributions from British Loyalists and continental Africans among different Bahamian islands. American Journal of Physical Anthropology, 146, 594-608. doi:10.1002/ajpa.21616

\section{Appendix}

Reference data were selected according to SNP assignment from YSearch database: (http://www.ysearch.org) and public projects of FTDNA

http://www.familytreedna.com/public/Haplogroup_A/default.as px? section=yresults

http://www.familytreedna.com/public/Chaplogroup/default.asp $\mathrm{x}$ ?section=yresults

http://www.familytreedna.com/public/HaplogroupE1andE/defa ult.aspx?section=yresults

http://www.familytreedna.com/public/E1bla/default.aspx?secti on=yresults

http://www.familytreedna.com/public/E3b/default.aspx?section $=$ yresults

http://www.familytreedna.com/public/G-YDNA/default.aspx?s ection=yresults

http://www.familytreedna.com/public/YHaploGroupH/default.a spx?section=yresults

http://www.familytreedna.com/public/yDNA_I1/default.aspx?s ection=yresults

http://www.familytreedna.com/public/I2nosubcladeM170P215/ default.aspx?section=yresults
http://www.familytreedna.com/public/I2aHapGroup/default.asp $\mathrm{x}$ ? section=yresults

http://www.familytreedna.com/public/Y-DNA_J/default.aspx?s ection=yresults

http://www.familytreedna.com/public/Y-Haplogroup-L/default. aspx? section=yresults

http://www.familytreedna.com/public/N\%20Y-DNA\%20Projec t/default.aspx? section=yresults

http://www.familytreedna.com/public/o3/default.aspx?section= yresults

http://www.familytreedna.com/public/R1Asterisk/default.aspx? section=yresults

http://www.familytreedna.com/public/R1aY-Haplogroup/defaul t.aspx?section=yresults

http://www.familytreedna.com/public/Y-Haplogroup-K2/defaul t.aspx?section=yresults Walk through the Y (International Project)

http://www.familytreedna.com/faq/answers/default.aspx?faqid= 27\#1324

https:/gap.familytreedna.com/media/docs/2010-FTDNA-TK.p df 